\title{
Absolute phase uncertainty in sinusoidal grating detection
}

\author{
JAMES H. HOWARD, JR, and KEVIN H. RICHARDSON \\ The Catholic University of America, Washington, D.C.
}

\begin{abstract}
Spatial phase plays an important role in the characterization of images and other visual patterns. Despite this, relatively few experiments have investigated the role of phase per se in human vision. Recent studies by Kersten (1983) and Burgess and Ghandeharian (1984) have shown that human observers are more sensitive to sinusoidal grating patterns when they have prior knowledge of the pattern's absolute phase than when they do not. They concluded that observers act as phase-sensitive detectors at least some of the time. Two forced-choice sinusoidal grating detection experiments are reported here which extend these results. Absolute signal phase was either held constant or varied randomly across trials. On half of the random-phase trials, observers were shown a sinusoidal grating cue that revealed the absolute phase of the test signal for that trial. There were three major findings. First, detection performance in both experiments was substantially better when phase information was provided than when it was not. This is consistent with previous findings. Second, information about signal phase was provided equally effectively by holding phase constant over all trials within a testing block (as in the constantphase conditions) or by providing an explicit phase cue $250 \mathrm{msec}$ before each trial. Third, a phase cue presented $250 \mathrm{msec}$ after the test pattern offset led to performance levels intermediate between the superior constant-phase condition and the uncued random-phase condition. In other words, observers were able to use phase information even when it was presented in a postcue. The findings are discussed in terms of alternative phase-sensitive detection models.
\end{abstract}

For many years psychophysicists have been interested in the ability of human perceivers to detect visual signals in noise. In a traditional visual detection task, observers are required to judge whether a well-specified visual target, such as a sinusoidal grating pattern, is present on each of a series of trials. Previous research has shown that a wide range of signal and subjective factors can influence the ability of an observer to detect even these simple targets. For example, signal parameters, such as spatial frequency, spatial extent, spatial location, temporal duration, orientation, and phase, are important (Graham, 1985), as are subjective factors, such as condition of dark adaptation, age, and the observer's prior knowledge of the signal. The experiments reported in this paper investigated the role of absolute signal phase and the observer's prior knowledge of phase in the detection of sinusoidal grating patterns embedded in a noise background. In this context, absolute phase refers to the alignment of the sinusoidal pattern (the location of light bands or peaks) within a viewing field.

\section{The Importance of Phase}

It is obvious that spatial phase plays an important role in the characterization of images and other visual patterns.

This research was supported by a contract from the Perceptual Sciences Program of the Office of Naval Research. The authors thank Alice J. $O$ 'Toole and Darlene V. Howard for their comments on an earlier version of the manuscript and Jeff Raver for his contribution to the work Requests for reprints should be addressed to the first author at Department of Psychology, Catholic University, Washington, DC 20064.
For example, without phase information, a simple target such as disk or square cannot be localized in an image. In their well-known paper on the importance of phase, Oppenheim and Lim (1981) demonstrated that in a Fourier representation of images, spectral phase alone can often lead to a recognizable reconstruction of the image when an analogous reconstruction from the spectral magnitude alone does not.

Despite the significance of phase in visual signals, relatively few experiments have investigated the role of phase per se in human vision. Research on spatial summation in the detection of complex signals has revealed evidence of phase sensitivity in some experiments (e.g., Lawden, 1983), but evidence of phase insensitivity in others (e.g., Koenderink \& van Doorn, 1980). Two experiments that have investigated the role of absolute rather than relative spatial phase have concluded that the human visual system acts as a phase-sensitive detector at least some of the time (Burgess \& Ghandeharian, 1984; Kersten, 1983). The experiments reported here extend this previous research on absolute phase sensitivity.

\section{Signal Detection Under Uncertainty}

In an early auditory experiment, Tanner and Norman (1954) found that pure-tone detection fell to chance levels when the test signal was shifted from a known frequency to another frequency without informing the listeners. Numerous follow-up experiments have demonstrated reduced detectability for pure tones when the frequency of the test tone is not predictable by the listener (Howard, 
O'Toole, Parasuraman, \& Bennett, 1984). A number of experiments have investigated an analogous frequency uncertainty effect in visual detection (Davis \& Graham, 1981). The major finding from these studies is that frequency uncertainty introduced by selecting the target signal randomly from a set of two or more spatial frequencies leads to a relatively small, but reliable, decrease in signal detectability relative to the single spatial frequency case. Uncertainty effects have also been established for other signal parameters. For example, Davis, Kramer, and Graham (1983) have shown comparable effects for spatial-position and spatial-frequency uncertainty but no effects for luminance-contrast uncertainty, and Ball and Sekuler (1981) have reported direction-uncertainty effects for motion detection. The spatial-position findings are also consistent with the large body of suprathreshold, locationuncertainty, reaction time experiments carried out by Posner and his colleagues (Posner, Nissen, \& Ogden, 1978).

Of greatest relevance to the present experiments, however, are two studies which have examined effects due to signal-phase uncertainty. In one experiment, Burgess and Ghandeharian (1984) presented observers with a pair of small, square, homogeneous Gaussian noise fields on each of a series of trials. One of the fields always contained a 4.6-cycle/degree (cpd) vertical sinusoidal grating added to the noise in random absolute phase. In other words, the absolute position of the sinusoid within the square was randomly determined on each trial and was therefore unpredictable. The observer's task was to make a forced-choice judgment indicating which of the two noise fields contained the pattern on each trial. A reference pattern, which contained a noise-free copy of the sinusoidal test signal for that trial, was also provided below each noise field. Two testing conditions were investigated. For test blocks with phase information, the reference signal was presented at the actual phase of the test signal, whereas for test blocks without phase information, the reference sinusoid was always presented with zero absolute phase. Hence, equivalent information was provided about the spatial frequency and spatial extent of the target under both testing conditions, but only the phaseinformation blocks provided any information about the phase of the test signal. Their results showed a clear detectability advantage when phase information was presented. This finding demonstrates that absolute phase plays a role in the detection of spatial-frequency gratings and that phase uncertainty can lead to decreased performance, as does uncertainty about other signal parameters such as spatial frequency and/or location.

In a similar experiment, Kersten (1983) examined the detectability of brief-duration sinusoidal gratings under fixed-and random-phase conditions. In the fixed-phase, or phase-certain, condition, signal phase was predictable, since it was held constant over trials and since a reference sinusoid preceded each forced-choice trial to illustrate the absolute phase of the target. On the other hand, in the random-phase, or phase-uncertain, condition, sig- nal phase was not predictable, since it varied randomly at $45^{\circ}$ intervals over the full period of the sinusoid. Kersten's findings revealed phase-uncertainty effects for lowfrequency $(.5$-cpd) stationary sinusoids embedded in noise, but no uncertainty effects when the same targets were presented without noise or when they were drifted slowly on the screen. Overall, the conclusions from this study support those of Burgess and Ghandeharian (1984) in demonstrating phase-uncertainty effects under "standard" detection-testing conditions.

\section{Uncertainty Reduction in Signal Detection}

Given that signal parameter uncertainty can lead to decreased detectability, a number of investigators have examined factors that lead to reduced signal uncertainty and, hence, improved detectability. For example, when Davis et al. (1983) used the pitch of a pure-tone cue to indicate the spatial frequency or spatial position of test targets under uncertain frequency or location conditions, detectability improved to match that of the alone, or fixedspatial-frequency (location), condition. Interestingly, this cuing effect occurred-albeit at a smaller magnitudeeven when the tonal cue was delayed for as long as $500 \mathrm{msec}$ after the offset of the test patterns (Graham, Kramer, \& Haber, 1985). Posner and his colleagues have also shown that for suprathreshold detection, benefits (i.e., speeded reaction times) accompany valid location cues whereas costs (i.e., retarded reaction times) accompany invalid cues (Posner et al., 1978).

Burgess and Ghandeharian's (1984) use of a simultaneous phase-reference pattern can be interpreted as supporting a similar uncertainty reduction for the phaseuncertainty case. These and other results (Graham et al., 1985) establish that providing supplementary knowledge regarding otherwise uncertain signal parameters can reduce or eliminate the uncertainty effects described in the previous section. The effectiveness of nonsimultaneous phase cues is the central issue investigated in the present study.

\section{Models of Signal Detection}

A common approach in the theoretical signal detection literature is to compare the empirical detection performance of human observers with that of theoretical ideal observers who respond optimally under a given set of theoretical assumptions. Although this approach has not been adopted in this paper, two traditional models that make different assumptions about spatial phase will be compared briefly to illustrate different possible roles of phase in visual detection. A more complete theoretical development may be found in Kersten (1983), Burgess and Ghandeharian (1984), or Caelli and Moraglia (1986).

On each trial in a two-alternative, forced-choice experiment (2AFC), the observer must choose between two image fields, one that contains a signal, $\mathrm{S}(x, y)$, added to uncorrelated noise, $\mathrm{N}(x, y), \operatorname{Isn}(x, y)=\mathrm{S}(x, y)+\mathrm{N}(x, y)$, and another which contains only a noise field, $\operatorname{In}(x, y)=$ 
$\mathrm{N}(x, y)$. Both models assume that this decision is based on a comparison of correlation integrals computed on each of the two image alternatives.

$$
\operatorname{Rsn}(a, b)=\iint \operatorname{Isn}(x, y) \mathrm{w}(x+a, y+b) d x d y
$$

and

$$
\operatorname{Rn}(a, b)=\iint \operatorname{In}(x, y) \mathrm{w}(x+a, y+b) d x d y,
$$

where $\mathrm{w}(x, y)$ is a weighting function, $a$ and $b$ are horizontal and vertical shifting factors, and the region of integration extends over the two spatial dimensions of the image fields. The two models make different assumptions about the weighting function, $w(x, y)$. Specifically, the cross-correlation model assumes that the signal parameters are known exactly, including signal phase, and hence the expected signal is used as the weighting function. For this reason, the cross-correlation model is sometimes referred to as a matched filter model. Since $\mathrm{w}(x, y)$ $=\mathrm{S}(x, y)$, the cross-correlation integral can be determined for the signal-plus-noise alternative by substitution

$$
\begin{aligned}
\operatorname{Csn}(a, b)= & \iint \operatorname{Isn}(x, y) \mathrm{S}(x+a, y+b) d x d y \\
= & \iint \mathrm{S}(x, y) \mathrm{S}(x+a, y+b) d x d y \\
& +\iint \mathrm{N}(x, y) \mathrm{S}(x+a, y+b) d x d y .
\end{aligned}
$$

In contrast, for the autocorrelation model, signal phase is not assumed to be known exactly, and hence the presented test image serves as the weighting function, $\mathrm{w}(x, y)=\operatorname{Isn}(x, y)=\mathrm{S}(x, y)+\mathrm{N}(x, y)$, and for the signalplus-noise alternative, the autocorrelation integral is

$$
\begin{aligned}
\operatorname{Asn}(a, b)= & \iint \operatorname{Isn}(x, y) \operatorname{Isn}(x+a, y+b) d x d y \\
= & \iint \mathrm{S}(x, y) \mathrm{S}(x+a, y+b) d x d y \\
& +\iint \mathrm{S}(x, y) \mathrm{N}(x+a, y+b) d x d y \\
& +\iint \mathrm{N}(x, y) \mathrm{S}(x+a, y+b) d x d y \\
& +\iint \mathrm{N}(x, y) \mathrm{N}(x+a, y+b) d x d y .
\end{aligned}
$$

In both cases, it is assumed that the forced-choice decision is based on a comparison of the correlation integrals for the two alternatives at zero shift (that is, when $a=b=0$ ). Furthermore, since the noise is assumed to be homogeneous (i.e., uncorrelated with the signal), the terms involving the cross-product of signal and noise will vanish over repeated trials. With these assumptions, both models discriminate between the signal-plus-noise and the noise-alone alternatives, since, in both cases, the correlation integral will be larger on the average for the signal- plus-noise alternative by an amount equivalent to the signal energy.

$$
\begin{aligned}
\operatorname{Asn}(x, y)-\operatorname{An}(x, y) & =\operatorname{Csn}(x, y)-\operatorname{Cn}(x, y) \\
& =\iint S^{2}(x, y) d x d y .
\end{aligned}
$$

The major difference between the phase-sensitive crosscorrelation model and the phase-insensitive autocorrelation model lies in the trial-by-trial variability of the correlation integrals. This difference is seen in a comparison of Equations 1 and 2 above. Specifically, the noise term is larger in the autocorrelation integral $\left[\iint \mathrm{N}^{2}(x, y) d x d y\right]$ than in the cross-correlation integral $\left[\iint \mathrm{N}(a, b)\right.$ $\mathrm{S}(a, b) d x d y$, and hence detection performance will be somewhat poorer in the autocorrelation case. As Burgess and Ghandeharian (1984) have pointed out, the essential distinction between the two models lies not in their relative sensitivity to the signal-both are sensitive to the signal energy-but in their ability to reject noise. This illustrates the difference between a simple coherent, or phase-sensitive, cross-correlation and an incoherent, or phase-insensitive, autocorrelation mechanism.

\section{EXPERIMENT 1}

As discussed above, previous results have suggested that human observers detect sinusoidal gratings in noise better when the signal phase is known than when it is not. This result is consistent with a coherent, or phasesensitive, detection mechanism. The present experiment extends this work by examining the ability of human observers to use phase information presented explicitly in phase cues that precede each trial. Three conditions were investigated. In the first, zero-phase condition, signal phase was predictable, since all signals within a testing block appeared at a constant absolute phase angle of $0^{\circ}$. In the second, random-phase condition, signal phase was not predictable, since phase angle was determined randomly on each trial. In the third, or cued/random-phase condition, phase was selected randomly on each trial but, unlike the random-phase condition, a cue was presented on each trial to inform the observer of the signal phase for that trial.

\section{Method}

Subjects. Five student and staff volunteers were paid $\$ 25$ to participate in the experiment. Each reported having normal or correctedto-normal acuity.

Apparatus. Image preparation, control of experimental events, and data analyses were carried out on a general-purpose laboratory computer (Digital VAX 11/750). This computer served as the controlling host for a Gould Imaging and Graphics IP8400 imageprocessing system that was used for on-line image processing, storage, and presentation. Participants were seated in a darkened, sound-attenuated room and viewed the test imagery on a 12-in. $(30.5-\mathrm{cm})$ diagonal, monochrome monitor (Ikegami Model PM14$3 \mathrm{H})$. Standard raster frequencies and an interlaced $30-\mathrm{Hz}$ frame rate were used with a display resolution of $512 \times 5128$-bit pixels. With the observers sitting at a viewing distance of $122 \mathrm{~cm}$, each pixel 
subtended a visual angle of $0.345 \mathrm{mrad}$ ( $1.19^{\prime}$ of arc). Participants entered their responses on a standard terminal keyboard, and graphic feedback was displayed at the center of the monitor to indicate the location of the correct pattern for that trial (either $<$ for left target or $>$ for right target). All test stimuli were passed through an intensity transform look-up table in the image processor to achieve a linear mapping of gray level onto luminance. The monitor was adjusted to have a full-range (0-255 gray levels) contrast ratio of $.59[(\mathrm{~L} \max -\mathrm{Lmin}) /(\mathrm{L} \max +\mathrm{Lmin})]$. Photometric calibrations were carried out using a Photo Research Litemate spot photometer.

Stimuli. Noise fields were generated by randomly sampling pixel values from a Gaussian distribution with a gray-level mean of 127 and a standard deviation of 40 gray levels. The $32 \times 32$ pixel noise fields were examined statistically and stored on disk for selection and use during the experiment. Two noise fields were sampled randomly on each trial, and a zero-mean vertical sinusoidal grating was added to one of the two. The sinusoid had a period of 17 pixels (frequency of $2.97 \mathrm{cpd}$ ), and the test fields (noise alone and signal plus noise) had a space average luminance of $162 \mathrm{~cd} / \mathrm{m}^{2}$. The test fields were positioned on either side of the screen center with a field separation of 31 pixels. Thus, at the viewing distance used, each field subtended $.63^{\circ}$ of visual angle and the entire display (both fields and gap) subtended $1.90^{\circ}$ of visual angle. The two-field test pattern for each trial, which required one video frame to display, remained in view for $266 \mathrm{msec}$.

Procedure. The experiment began with a preliminary session to determine individual-observer testing levels for the experiment. In this session, six 80-trial blocks were completed in which the amplitude of the sinusoidal target was adjusted by an up-and-down staircase to yield a $70 \%$ correct detection level. Individual testing levels were then based on $150 \%$ of the mean signal amplitude for that observer over the last five preliminary blocks. The noise levels remained constant. This procedure simplified the task relative to threshold levels and resulted in peak sinusoidal amplitudes of 9, $11,12,14$, and 15 gray levels for the 5 observers, respectively.

Following this, each observer participated in three testing sessions on separate days. Each of the three testing sessions involved six 100-trial blocks ( 20 practice and 80 test trials), two each of the zero-, random-, and cued/random-phase conditions. All target signals in a zero-phase block were presented with a phase angle of $0^{\circ}$. In other words, the positive-slope, zero-crossing point of the sinusoid occurred at the leftmost boundary of the noise field. On the other hand, for both the random and the cued/random blocks, the sinusoidal phase angle varied randomly on each trial. Since the instrumentation limited phase shifts to discrete, one-pixel steps, nine different phase values were used, ranging from $0^{\circ}$ to $180^{\circ}$ in $20^{\circ}$ steps. On practice trials the signal amplitude was reduced gradually from an initial high-contrast level to the final testing level. The signal-to-noise ratio remained constant over the remaining 80 test trials. The order of the zero, random, and cued/random conditions was determined randomly for each session.

Trials in the zero- and random-phase blocks began with the presentation of a centrally located fixation cross, which remained in view for approximately $500 \mathrm{msec}$. The fixation point was erased and, after a 250-msec delay, the two test fields were displayed for $266 \mathrm{msec}$. For the cued/random blocks, a centrally located, $32 \times 32$ pixel, high-contrast, sinusoidal grating at the randomly selected phase for that trial served as the fixation. In other words, the precue was exposed for $500 \mathrm{msec}$ and was erased $250 \mathrm{msec}$ before the test patterns were presented. The observers were told before the experiment that they were to determine which of the two boxes of "speckle" contained a pattern of light and dark "bars." They were also told to concentrate carefully on the cue provided on the cued/random blocks, since it was "an example of the pattern they would be looking for," and that this example should be "very helpful" to them. No specific instructions were given concerning signal phase.

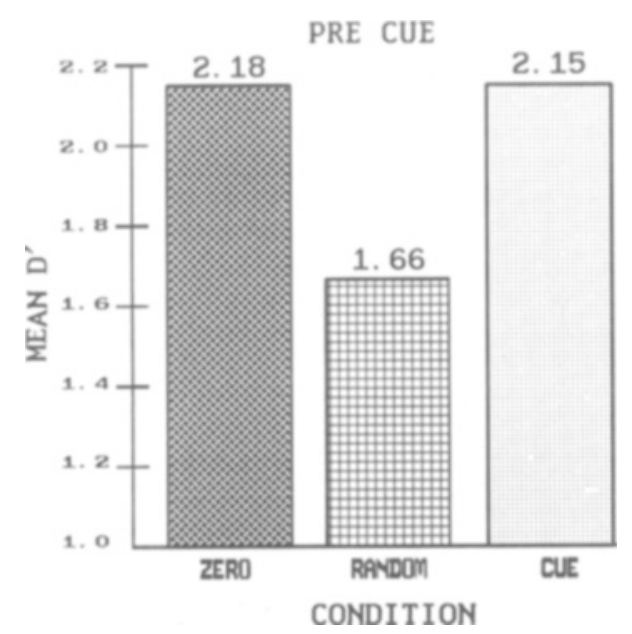

Figure 1. Mean response-bias free performance $\left(d^{\prime}\right)$ for the zero-, random-, and cued/random-phase testing conditions of Experiment 1.

Observers entered their responses by pressing a key, and feedback immediately followed the response. The next trial began when the observer pressed the space bar on the keyboard. Each session required about $1 \mathrm{~h}$ to complete.

\section{Results and Discussion}

Response-bias free estimates of sensitivity $\left(d^{\prime}\right)$ were determined for each testing block and observer from the corresponding hit (correctly responding left) and false-alarm (incorrectly responding left) rates (Macmillan \& Kaplan, 1985). The mean sensitivity collapsed across observer and block is shown in Figure 1 for the zero-, random-, and cued/random-phase testing conditions. Examination of Figure 1 reveals a large effect of condition, with the random-phase trials producing considerably lower sensitivity (mean $d^{\prime}=1.66$ ) than either the zero- (mean $d^{\prime}$ $=2.18$ ) or the cued/random-phase (mean $d^{\prime}=2.15$ ) trials. These results were submitted to a two-way (block $x$ condition) analysis of variance with repeated measures on both factors. This revealed a significant main effect of condition $[F(2,8)=8.29, p<.025, M S \mathrm{e}=1.28]$, with neither the main effect of block $[F(2,8)<1.0]$ nor the block $\times$ condition interaction $[F(4,16)=1.24]$ reaching statistical significance.

A post hoc analysis of the condition effect was carried out using Duncan's new multiple range test. This revealed that the observers were more sensitive when absolute phase was predictable (zero and cued/random conditions) than when it was not predictable (random condition), and that they were equally sensitive under the zero-phase and cued/random-phase conditions. A similar pattern occurred for each of the observers, as may be seen in the individual data tabulated by condition in Table 1 .

These findings are consistent with those of Kersten (1983) and Burgess and Ghandeharian (1984) in arguing for a coherent or phase-sensitive detection mechanism. It is clear that observers are more sensitive when phase 
Table 1

Individual Observer Data (Mean $d^{\prime}$ ) by Condition, Experiment 1

\begin{tabular}{ccccc}
\hline & \multicolumn{4}{c}{ Phase Condition } \\
\cline { 2 - 5 } Observer & Zero & Random & Cued/Random & Mean \\
\hline 1 & 1.71 & 1.66 & 2.01 & 1.79 \\
2 & 1.64 & .99 & 1.42 & 1.35 \\
3 & 2.68 & 2.39 & 2.94 & 2.67 \\
4 & 3.06 & 1.91 & 2.53 & 2.50 \\
5 & 1.81 & 1.34 & 1.85 & 1.67 \\
Mean & 2.18 & 1.66 & 2.15 & 2.00 \\
\hline
\end{tabular}

information is provided than when it is not. Furthermore, observers are equally good at using phase information whether it is provided by cuing on a trial-by-trial basis, as in the cued/random condition, or by holding the phase constant over trials, as in the zero-phase condition. This indicates that observers are able to adjust their signal template or matched filter in response to phase information provided on a trial-by-trial basis. Since precise gray-level information is required for the effective use of a matched filter, these findings also suggest that observers are able to maintain a relatively detailed representation of the signal cue throughout the 250 -msec period between the cue offset and test signal onset.

\section{EXPERIMENT 2}

Although it is clear from the results of Experiment 1 that phase information is important, the experiment did not address the question of where or at what level in processing the phase information is being used. For example, it is possible that phase is important in early processing and that the phase of the target must be known before the test patterns are presented (as in prior or simultaneous cue presentation). In this view, the phase information could be incorporated into a phase-calibrated "input-selection" filter that produces a greater crosscorrelation response to the signal-plus-noise field than to the noise-alone field. On the other hand, phase information may become important only later in processing after a higher order perceptual representation of the test fields has been constructed.

These alternatives were examined in Experiment 2 by extending the method of Experiment 1 to investigate the effectiveness of a postcue in reducing phase uncertainty. As in the first experiment, three conditions are used: zero-, random-, and cued/random-phase. Unlike the first experiment, however, in this experiment the phase cue was presented following the offset of the two-field test pattern. If the postcue was effective in reducing phase uncertainty, then the early-processing or input-selection model could be rejected.

\section{Method}

Subjects. Five student and staff volunteers participated in the experiment. None had participated in Experiment 1, and all had normal or corrected-to-normal acuity. As in the first experiment, the observers were paid a base rate of $\$ 5$ for each of the five sessions.
Apparatus and Stimuli. The apparatus and stimuli were identical to those used in Experiment 1.

Procedure. With the exception of the use of a postcue rather than a precue, the procedure was identical to that used in Experiment 1. As in the uncued conditions of Experiment 1, each postcue trial began with a centrally displayed fixation cross which remained in view for approximately $500 \mathrm{msec}$. The fixation was then erased and, after a 250-msec delay, the test patterns were displayed for $266 \mathrm{msec}$. After another 250-msec delay, the postcue was displayed at the central fixation point and remained in view for approximately $500 \mathrm{msec}$. Thus, each postcue trial was approximately $750 \mathrm{msec}$ longer than the precue trials of Experiment 1. For this experiment, preliminary testing resulted in peak sinusoidal testing amplitudes of $7,8,10,14$, and 14 gray levels for the 5 observers, respectively.

\section{Results and Discussion}

Response-bias free estimates of sensitivity $\left(d^{\prime}\right)$ were determined for each testing block and observer from the corresponding hit and false-alarm rates. The mean sensitivity collapsed across observer and block is shown in Figure 2 for the zero-, random-, and cued/random-phase testing conditions. Visual inspection of these data reveals that the random-phase trials produced considerably lower sensitivity (mean $d^{\prime}=1.13$ ) than the zero-phase trials (mean $d^{\prime}=1.55$ ), whereas the cued/random-phase trials led to performance (mean $d^{\prime}=1.34$ ) that fell midway between the other two conditions. In other words, the postcue was effective in reducing phase uncertainty, but not as effective as holding phase constant as in the zero-phase condition. These data were analyzed by a two-way (block $x$ condition) analysis of variance with repeated measures on both factors. As in the first experiment, a significant main effect of condition was obtained $[F(2,8)=9.39$, $p<.01, M S \mathrm{e}=0.66]$ with no significant main effect of block or block $\times$ condition interaction.

A post hoc analysis of the condition effect (Duncan's new multiple range test) revealed greater sensitivity when absolute phase was predictable (zero and cued/random

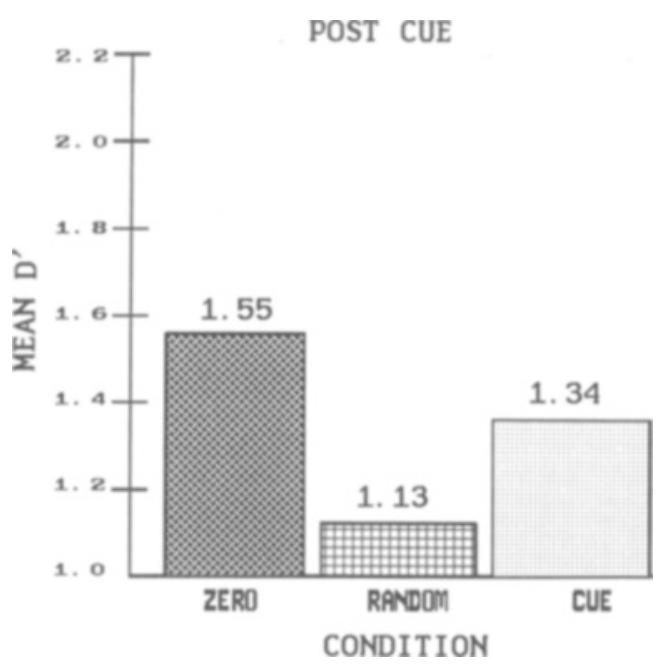

Figure 2. Mean response-bias free performance $\left(d^{\prime}\right)$ for the zero-, random-, and cued/random-phase testing conditions of Experiment 2. 
Table 2

Individual Observer Data (Mean $d^{\prime}$ ) by Condition, Experiment 2

\begin{tabular}{|c|c|c|c|c|}
\hline \multirow[b]{2}{*}{ Observer } & \multirow[b]{2}{*}{ Zero } & \multicolumn{2}{|c|}{ Phase Condition } & \multirow[b]{2}{*}{ Mean } \\
\hline & & Random & Cued/Random & \\
\hline 1 & 2.05 & 1.85 & 1.91 & 1.94 \\
\hline 2 & 1.29 & .95 & .89 & 1.04 \\
\hline 3 & 1.29 & .50 & 1.13 & .97 \\
\hline 4 & 2.23 & 1.84 & 2.16 & 2.07 \\
\hline 5 & .88 & .51 & .61 & .67 \\
\hline Mean & 1.55 & 1.13 & 1.34 & 1.34 \\
\hline
\end{tabular}

conditions) than when it was not predictable (random condition), and greater sensitivity under the zero-phase condition than under the cued/random-phase condition. As before, a similar pattern occurred for each of the $5 \mathrm{ob}-$ servers, ruling out the possibility that the intermediate performance level obtained for the cued/random condition reflects averaging bias. The individual data are tabulated by condition in Table 2 .

These findings argue against the early-selection model. If prior information about signal phase were required for observers to use a phase-sensitive detection mechanism, then a phase postcue would not have been effective in reducing uncertainty. This finding can be explained by a broad class of late-selection models which argue that the cue information becomes important relatively late in processing. ${ }^{1}$

\section{GENERAL DISCUSSION}

There are three main findings in the present study. First, phase information is important in sinusoidal grating detection. Performance in both experiments was substantially better when phase information was provided than when it was not. Second, information about signal phase can be provided equally effectively by holding phase constant over all trials within a testing block (as in the zerophase conditions) or by providing an explicit phase cue on each trial (as in the cued/random conditions). Third, observers are able to use phase information even if it is provided $250 \mathrm{msec}$ after the offset of the test signals. Some implications of these findings will be considered below.

\section{Phase Is Important}

The results reported here are consistent with those of Burgess and Ghandeharian (1984) and Kersten (1983) in arguing for a phase-sensitive or coherent detection mechanism. However, both authors have argued that while phase-sensitive detection can occur, it need not always occur. For example, Kersten found little effect of prior phase information for noise-free or slowly moving sinusoidal signals (Kersten, 1983), and Burgess and Ghandeharian (1984) found that human detection abilities exceeded that of an ideal phase-insensitive observer only for relatively limited regions of the psychophysical curve. The present results are consistent with this, and support a twomechanism detection strategy. Observers may have used a phase-sensitive mechanism whenever it was possible to do so (i.e., in the zero and cued/random conditions) and a phase-insensitive mechanism in the other case (i.e., random condition).

This explanation for the phase-uncertainty effect is similar to the multichannel theories invoked to account for spatial-frequency-uncertainty effects (see Graham, 1985). According to these theories, detection performance is better when prior frequency information is available because observers need only monitor selected, probable input channels and therefore are less likely to experience a false alarm on one of the noise-only channels (Yager, Kramer, Shaw, \& Graham, 1984). By a similar argument, phasesensitive detection mechanisms are more effective in noise-rejection than are phase-insensitive mechanisms. In other words, it is easier to reject spurious, pattern-like correlations within the noise when one knows exactly what to look for. Both the phase-uncertainty and frequencyuncertainty effects are well described by noise-limited models of this sort.

\section{The Representation of Supplementary Phase Information}

In the cued/random conditions investigated here, phase cues either preceded or followed the test fields, and phase angle varied randomly on a trial-by-trial basis. Our results indicated that for both the precue condition of Experiment 1 and the postcue condition of Experiment 2, these varying, nonsimultaneous cues were effective in reducing phase uncertainty. This implies that observers have considerable flexibility in their use of phase information, and in particular, it can be argued that the matched filter used in cross-correlation can be adapted within the time frame of a single trial. Furthermore, it implies that the matched filter and/or test fields must be retained for a brief time in some form of memory or internal representation.

This raises an important question regarding the form of internal representation used in cross-correlation detection. Are literal gray-level images retained or is the representation more descriptive and higher order? In a recent paper, Caelli and Moraglia (1986) have distinguished two broad classes of cross-correlation models pertinent to this issue. On the one hand, there are direct models, which assume that the cross-correlations are carried out directly between the matched filter (i.e., the expected signal) and the test fields. These traditional models obviously require that relatively literal gray-level information be retained. On the other hand, there are also indirect cross-correlation models in which the correlations are carried out between some decomposition of the expected signal and a similar encoding of the test fields. A wide range of possibilities exists for such a processed representation, including the outputs of spatial scale or frequency analyzers and other primitive edge or "primal sketch" elements, as proposed by Marr (1982) and others. What is important here is that the internal representation required by these models is higher order and more descriptive than the gray levels assumed in traditional 
cross-correlation models. For the case of the sinusoidal gratings investigated here, a higher order representation may simply be a description of the relative locations of light and dark bands within the square signal field. Although alternative, gray-level representations, such as retinal afterimages, cannot be ruled out by the present data, observers disclaim any awareness of persisting afterimages. This issue will be considered further in the following section.

\section{The Evidence for Late Processing of Phase}

Our finding that postcues are effective in reducing phase uncertainty argues against an early-selection or input-filter model of cross-correlation. In other words, it is not necessary to know literally what signal you are looking for before the test fields are presented. This evidence for the late processing of phase information is consistent withBurgess and Ghandeharian's (1984) conclusion that "suprathreshold detection and decision tasks are performed at a high (cognitive) level in the brain" (p. 904). It is also consistent with what Graham and her colleagues have termed a conscious-rejection strategy for rejecting false positives from nontarget channels. According to this view, the observer "consciously realizes that some of what is seen corresponds to stimuli that are definitely not present ... and so consciously ignores these percepts when making his or her response" (Graham et al., 1985, p. 277). Informally, some observers in the present study did report occasionally experiencing and then rejecting such phantoms. A late-selection, conscious-rejection model of this sort is consistent with the large phaseuncertainty effects reported here. Since the hypothesized phantom signals result from spurious correlations within the noise, their location or phase would vary randomly, making them very difficult to discriminate from "true", signals of uncertain phase. Although plausible, it is too early to embrace this theory on the basis of the present data.

\section{REFERENCES}

Ball, K., \& Sekuler, R. (1981). Cues reduce direction uncertainty and enhance motion detection. Perception \& Psychophysics, 30, 119-128.

Burgess, A., \& GHandeharian, H. (1984). Visual signal detection: I. Ability to use phase information. Journal of the Optical Society of America, 1, 900-905.

CaElli, T., \& Moraglia, G. (1986). On the detection of signals embedded in natural scenes. Perception \& Psychophysics, 39, 87-95.

Davis, E. T., \& Graham, N. (1981). Spatial frequency uncertainty effects in the detection of sinusoidal gratings. Vision Research, 21, 705-712.
Davis, E. T., Kramer, P., \& Graham, N. (1983). Uncertainty about spatial frequency, spatial position, or contrast of visual patterns. Perception \& Psychophysics, 33, 20-28.

GraHAM, N. (1985). Detection and identification of near-threshold visual patterns. Journal of the Optical Society of America, 2, 1468-1482.

Graham, N., Kramer, P., \& Haber, N. (1985). Attending to the spatial frequency and spatial position of near-threshold visual patterns. In M. I. Posner \& O. S. M. Marin (Eds.), Attention and Performance $X I$ (pp. 269-284). Hillsdale, NJ: Erlbaum.

Howard, J. H., Jr., O'Toole, A. J., Parasuraman, R., \& Bennett, K. B. (1984). Pattern-directed attention in uncertain-frequency detection. Perception \& Psychophysics, 35, 256-264.

KeRSTEN, D. (1983). A comparison of human and ideal performance for the detection of visual patterns. Unpublished doctoral dissertation, University of Minnesota.

Koenderink, J. J., \& VAN DOORN, A. J. (1980). Spatial summation for complex bar patterns. Vision Research, 20, 169-176.

LAWDEN, M. C. (1983). An investigation of the ability of the human visual system to encode spatial phase relationships. Vision Research, 23, 1451-1463.

MaCmillan, N., \& Kaplan, H. L. (1985). Detection theory analysis of group data: Estimating sensitivity from average hit and false-alarm rates. Psychological Bulletin, 98, 185-199.

MARR, D. (1982). Vision. San Francisco: W. H. Freeman.

OPPENheim, A. V., \& LIM, J. S. (1981). The importance of phase in signals. Proceedings of the IEEE, 69, 529-541.

Posner, M. I., Nissen, M. J., \& OGden, W. C. (1978). Attended and unattended processing modes: The role of set for spatial location. In H. Pick, Jr., \& E. Saltzman (Eds.), Modes of perceiving and processing information (pp. 137-157). Hillsdale, NJ: Erlbaum.

TANner, W. P., \& Norman, R. Z. (1954). The human use of information: II. Signal detection for the case of an unknown signal parameter. Transactions of the IRE Professional Group on Information Theory, PGIT-4, 222-227.

Yager, D., Kramer, P., Shaw, M., \& Graham, N. (1984). Detection and identification of spatial frequency: Models and data. Vision Research, 24, 1021-1035.

\section{NOTE}

1. A substantial overall performance difference occurred between Experiments 1 (mean $d^{\prime}=2.00$ ) and 2 (mean $d^{\prime}=1.34$ ). Although this overall difference was not found to be statistically significant in a fourway mixed-design analysis of variance $[F(1,8)=3.05]$, the large difference between the two comparable zero and random conditions was somewhat surprising. To examine this further, an additional postcue experiment was carried out in which the testing levels were set to $175 \%$ of threshold rather than $150 \%$. The new experiment produced performance comparable to Experiment 1 in the zero and random conditions with an overall pattern identical to that observed in Experiment 2 (mean $d$ 's of $2.03,1.46$, and 1.59 for the zero, random, and postcue conditions, respectively). This suggests that the differences in overall level observed between Experiments 1 and 2 may be attributable to an overall set effect induced by the less effective postcue condition.

(Manuscript received January 22, 1987; revision accepted for publication July 2, 1987.) 